Communications in Contemporary Mathematics, Vol. 3, No. 4 (2001) 649-652

(C) World Scientific Publishing Company

\title{
ERRATUM TO: TIGHT CONTACT STRUCTURES ON LENS SPACES
}

\author{
JOHN B. ETNYRE \\ Department of Mathematics, Stanford University, Stanford, CA 94305, USA \\ etnyre@math.stanford.edu
}

The Proof of Lemma 3.6 in [1] is incorrect but all the main theorems in the paper are still correct. Lemma 3.6 can still be proven for the lens spaces $L(p, q)$ when $q=1$ or $p-1$. (We include the proof for these cases at the end of the paper since [2] used Lemma 3.6 when $q=p-1$.) However, the lemma may by bypassed in the essential arguments in [1]. Recall that the prime use of Lemma 3.6 was to show that we could assume that the generalized projective plane $D$ in $L(p, q)$ (i.e. the two skeleton) had at most $p$ elliptic points in its characteristic foliation. This is still true:

Lemma 1. We may (topologically) isotop $D$ in $L(p, q)$ so that $e_{+}(D) \leq p$.

Note that with this lemma in hand Theorem 4.3, Corollary 4.5 and Theorem 4.10 from [1] are true and their proofs are essentially unchanged.

Proof. We assume that we have isotoped $D$ so that $e_{+}(D)$ is minimal. Now if $e_{+}(D)>p$ then we derive a contradiction.

Recall that by choosing a point $x$ on the one skeleton $C$ of $L(p, q)$ we break $\partial D$ into $p$ intervals $B_{1}, \ldots, B_{p}$ using the $p$ points $x_{1}, \ldots, x_{p}$ on $\partial D$ that map to $x$ when $D$ is glued to $C$. We say that an interval $I$ on $\partial D$ is longer than $k$ if for any choice of $x$ the interior of the interval $I$ contains at least $k$ of the $x_{i}$ 's.

Note by [1, Remark 3.9] we know that if we look at an outermost hyperbolic singularity on $D$ (i.e. one whose unstable manifolds separate off a disk $\Delta$ containing one elliptic point) then $\Delta \cap \partial D$ is longer than 1 . Now consider a hyperbolic point $h$ whose unstable manifolds separate off a disk $\Delta$ from $D$ that contains two elliptic points and one hyperbolic point $h^{\prime}$. We claim that $I=\Delta \cap \partial D$ is longer than 2 . To see this let $I=I_{c} \cup I_{m} \cup I_{a}$ where $I_{m}$ and the unstable manifolds of $h^{\prime}$ bound a disk on $D$. We know that $I_{m}$ is longer than 1 so if $I$ is not longer than 2 then both $I_{c}$ and $I_{a}$ are not longer than 1 . Moreover, in this situation it is not hard to see that the intervals $I_{c}$ and $I_{a}$ are disjoint when mapped to $C$. Thus just as in [1, Fig. 9] we may construct an overtwisted disk by extending the unstable manifolds of $h$ and $h^{\prime}$ across $C$. 
The above argument generalizes to prove: if $h$ is a hyperbolic point whose unstable manifolds separate off a disk $\Delta$ containing a linear graph in the characteristic foliation containing $k$ elliptic points, then $\Delta \cap \partial D$ is longer than $k$. There is one complication in this case that is not seen above. If $h^{\prime}$ is the hyperbolic point in $\Delta$ whose unstable manifolds separate off a disk $\Delta^{\prime}$ containing $k-1$ elliptic points, then by induction on $k$ we can assume that $\Delta^{\prime} \cap \partial D$ is longer than $k-1$. So if our claim is not true then $I_{c} \cup I_{a}=S \cap \partial D$ is not longer than 1 and $I_{c}$ and $I_{a}$ are disjoint when mapped to $C$, where $S=\overline{\Delta \backslash \Delta^{\prime}}$. As above we would like to say that we can construct an overtwisted disk as in [1, Fig. 9]. Unfortunately one of the intervals, say $I_{a}$, might not limit to a single elliptic point when pushed across $C$ (strangely enough this does not happen in the situation above), but if this happens the other interval will limit to a single elliptic point when pushed across $C$. So if $K$ is the union of all leaves in $D_{\xi}$ that intersect $I_{c}$ and end at a fixed elliptic point, then we can find a disk $B$ close to $S \cup K$ such that $B \cap C$ is a neighborhood of $I_{a}$ on $C$ and $B$ contains a single hyperbolic point $h$ whose unstable manifolds intersect $C$ in $\partial I_{a}$. Note we might have $B \cap D \neq \emptyset$ but then we may (topologically) isotope $D$ keeping $C$ fixed so that it has the same number and type of singularities and is disjoint from $B$ (away from $C$ ). Now as in the proof of [1, Lemma 3.8] we may use this disk $B$ to decrease $e_{+}(D)$ by $p$ (contradicting the minimality of $e_{+}(D)$ ). Combining this argument with the one in the proof of [1, Theorem 4.6] one may easily remove the word "linear" from the above statement.

We now know that $e_{+}(D) \leq p$ since the total "length" of $\partial D$ is $p$.

Theorem 4.6 (and hence Corollary 4.7 and Theorems 4.8 and 4.9) in [1] follow from the above proof as follows: Note that under the hypothesis of Theorem 4.6 there must be $p$ elliptic and $p-1$ hyperbolic singularities in $D_{\xi}$. If we take an outermost hyperbolic point $h$ then its unstable manifolds separate $D$ into two disks $\Delta_{0}$ and $\Delta_{1}$ and by the remark at then end of the above proof $I_{0}=\Delta_{0} \cap \partial D$ is longer than 1 while $I_{1}=\Delta_{1} \cap \partial D$ is longer than $p-1$. Now take a point $x$ on $C$ that is in the intersection of $C$ with the unstable manifolds of $h$. The interior of $I_{0}$ contains at least 1 of the points $x_{i}$ while the interior of $I_{1}$ contains at least $p-1$ of the points $x_{i}$. That means that at least $p$ of the $x_{i}$ 's are accounted for on the interiors of the respective intervals but one of the $x_{i}$ 's in on the boundary of both intervals. This contradicts the fact that there are only $p, x_{i}$ 's. Thus there can be no tight contact structure satisfying the hypothesis of Theorem 4.6.

We have now demonstrated that the main theorems in [1] are correct; but, in order to repair a gap in [2] caused the incorrect proof of [1, Lemma 3.6] we show that the lemma is indeed correct in the cases relevant to [2].

Proof of [1, Lemma 3.6] for $\boldsymbol{q}=\mathbf{1}$ or $\boldsymbol{p}-\mathbf{1}$. We will show how to isotope $D$ to a disk $D^{\prime}$ with transverse boundary in $\partial V_{1}$, whose graph of singularities relates to $D$ 's as shown in [1, Fig. 2]. Since the graph of singularities in $D_{\xi}$ must be a tree, a sequence of such moves will clearly yield the conclusion of the lemma. 
Assume that part of the graph of singularities in $D_{\xi}$ is as shown on the left hand side of [1, Fig. 2] and let $h$ be the hyperbolic singularity whose stable separatrix we wish to move. The unstable separatrices of $h$ cut $D$ into two pieces: one, $\Delta$, containing only one elliptic singularity $e$ and one, $D \backslash \Delta$, containing all the other singularities. Let $U$ be a (closed) neighborhood, in $V_{1}$, of $\partial V_{1} \cup \Delta$ for which $U \cap D$ contains only the singularities $h$ and $e$. We may assume that $U$ is diffeomorphic to $\left(\partial V_{1}\right) \times[0,1]$ and that the characteristic foliations on both boundary components of $\partial U$ are non singular. Moreover we need $D \cap U$ to have transverse boundary. Here is where we must assume that $q=1$ or $p-1$ since $(\partial U) \backslash\left(\partial V_{1}\right)$ will naturally have four singularities that need to be canceled. We would like to do this cancelation in the complement of $D \cap U$ so as to keep it transverse. By taking $V_{0}$ to be a sufficiently small neighborhood of its core $C$ when $q=1$ or $p-1$ we may achieve this (as the reader my easily verify by looking at the monodromy on $\partial D$ induced by the characteristic foliation of $\left.\partial V_{1}\right)$. Now let $A=D \cap U, D^{\prime}=\overline{D \backslash A}, c=\partial D^{\prime}$ and $x$ be the intersection of $c$ with the stable seperatrix of $h$ that we wish to move. Note there is a region $R \subset c$ such that if $D_{\xi}^{\prime}$ is glued to $A_{\xi}$ via a diffeomorphism $\psi: c \rightarrow c$ that takes $x$ into $R$ the resulting singular foliation is as seen on the right hand side of [1, Fig. 2]. We now show how to "realize" such a diffeomorphism by isotoping $A$.

Let $T^{\prime}$ be the boundary component of $U$ that lies in the interior of $V_{1}$. Since the characteristic foliation of $T^{\prime}$ is non singular and contains no leaves parallel to $c$ (since $\xi$ is tight) we may use $T_{\xi}^{\prime}$, thought of as a flow, to define a Poincaré return map $\phi: c \rightarrow c$. We can assume that $\phi$ has irrational rotation number since by isotoping $T^{\prime}$ in the neighborhood of a meridional curve (away from $D$ ) we will change $\phi$. During this isotopy the rotation numbers for the corresponding $\phi$ 's will change and thus at some point be irrational.

If we cut $T^{\prime}$ along $c$ we get annulus $A^{\prime}$. Gluing one boundary component of $A^{\prime}$ to $c \subset A$ and the other to $c \subset D^{\prime}$ and rounding corners we have a new meridional disk $D_{1}$ whose characteristic foliation is $D_{\xi}^{\prime}$ glued to $A_{\xi}$ via $\phi$. (Note that using Makar-Limanov's corner rounding method [3] one can round both corners above without altering the topological type of the characteristic foliation.) It is useful to think of $D_{1}$ as obtained from $D$ by pushing part of the interior of $D$ once around $V_{1}$.

If $\phi$ does not take $x$ into $R$ then we do not have the desired characteristic foliation. But since $\phi$ has irrational rotation number the orbit of $x$ under $\phi$ is dense in $c$. Thus there is some power, say $n$, of $\phi$ that will take $x$ into $R$. If we take $n$ disjoint copies of $T^{\prime}$ then we can do the above procedure using all $n$ copies of $T^{\prime}$ to obtain a disk $D_{n}$. Of course the characteristic foliation on $D_{n}$ will not be exactly $D_{\xi}^{\prime}$ glued to $A_{\xi}$ via $\phi^{n}$ since the Poincaré return maps on the copies of $T^{\prime}$ are not exactly $\phi$. But if we take the copies of $T^{\prime}$ to be sufficiently close to $T^{\prime}$ then the gluing map will be close enough to $\phi^{n}$ to still take $x$ into $R$. Thus $D_{n}$ will be the desired new meridional disk whose characteristic foliation is related to $D$ 's as seen in [1, Fig. 2]. 


\section{Acknowledgments}

The author thanks R. Gompf for pointing out the mistake in [1].

\section{References}

[1] J. Etnyre, Tight contact structures on lens spaces, Commun. Contemp. Math. 2(4) (2000) 559-577.

[2] S. Ko, More about tight contact structures on lens spaces, Dissertation, UC Berkeley (2000).

[3] S. Makar-Limanov, Tight contact structures on solid tori, Trans. Amer. Math. Soc. 350 (1998) 1013-1044. 